\title{
Evaluation for Granulomatous Inflammation on Fine Needle Aspiration Cytology Using Special Stains
}

\author{
Muhammad Mudassar Majeed and Mulazim Hussain Bukhari \\ Department of Pathology, King Edward Medical University, 26 MOF, GOR-3, Shahdman-Lahore 54000, Pakistan \\ Correspondence should be addressed to Mulazim Hussain Bukhari, drmhbukhari@yahoo.com
}

Received 21 December 2010; Accepted 3 May 2011

Academic Editor: Darshana Jhala

Copyright ( $) 2011$ M. M. Majeed and M. H. Bukhari. This is an open access article distributed under the Creative Commons Attribution License, which permits unrestricted use, distribution, and reproduction in any medium, provided the original work is properly cited.

\begin{abstract}
Background. Tuberculosis is the commonest infectious disease in the developing world. Many diagnostic tests are devised for its detection including direct smear examination. This study was designed to determine the frequency of cases positive for AFB and positive for fungus in patients diagnosed to have granulomatous inflammation on Fine Needle Aspiration Cytology using special stains. Materials and Methods. A descriptive cross-sectional survey was done on 100 cases of granulomatous inflammation consistent with tuberculosis diagnosed on fine needle aspiration cytology at the Department of Pathology, King Edward Medical University, Lahore. After reporting granulomatous inflammation on Hematoxylin \& Eosin staining of aspirates from FNAC, some unstained slides were subjected to special stains, like ZN, GMS, and PAS. Cases positive for AFB on ZN stain and fungus on GMS/PAS were noted down along with their frequency and percentages. Results. Forty-four cases (44\%) of AFB positive smears were reported in granulomatous inflammation while only $5 \%$ cases of fungus were reported down. Cervical lymph nodes were the most commonly involved site (87\%), and females were affected more (62\%) than males. Most cases of AFB-positive smears were associated with caseation necrosis $(93 \%)$. Conclusion. Special stains should be done on all granulomatous inflammation cases seen on FNAC for confirmation of TB and ruling out other infectious causes.
\end{abstract}

\section{Introduction}

Tuberculosis is playing havoc throughout the world, and this is especially true for the developing countries. Every year 8 million new cases are seen and 2 million deaths occur because of Tuberculosis [1]. In Pakistan, the estimated incidence of Tuberculosis is $181 / 100000$ [2]. Tuberculosis (TB) carries a high risk of morbidity and mortality. TB has widespread involvement and rarely any tissue or organ is not involved by it. Most common is the pulmonary involvement [3] which has caused numerous deaths in the past. It can also involve the appendix [4], small and large intestine [5], skin [6], soft tissues, lymph nodes [7], genitourinary tract [8], and brain [9]. The dilemma does not end here and many other unusual organs are also involved [10].

The histology of TB is a characteristic showing granuloma formation by epithelioid histiocytes and Langhan's type Giant cells with or without caseation necrosis. This pattern is also preserved somehow in cytology specimens
[11]. Infectious causes most notably presenting with granulomatous inflammation is Mycobacterium Tuberculosis with a reported frequency of $59.4 \%$ [11] and fungal causes $[12,13]$ with a reported frequency of $20.4 \%$ [14]. Other common causes include Sarcoidosis [15], Wegener's granulomatosis [16], Actinomycosis [17], Crohn's diseases [18], Histoplasmosis [19], foreign body, and Langerhans cell histiocytosis [20].

Pertaining to a broad differential diagnosis, the diagnosis of tuberculosis remains a challenge. History and clinical examination are always very helpful. Many diagnostic tests are in practice. Every test has its own sensitivity and specificity and limitations. The commonly performed tests include examination of sputum for Acid Fast Bacilli [21], Cultures for Mycobacterium tuberculosis [22], Fine Needle Aspiration Cytology (FNAC) [23], Biopsy, and PCR [24].

Fine Needle Aspiration Cytology is a minimally invasive and time-saving procedure, which helps in the diagnosis of number of diseases especially in palpable nodules of 
breast, lymph node disorders [25], thyroid [26], and palpable skin and subcutaneous nodules. It has become very popular nowadays among physicians and surgeons because of its benefits. In clinical practice, it helps them to reach a diagnosis or at least plan beforehand the proper management of the patient. As we have already discussed that granulomatous inflammation is not diagnostic of $\mathrm{TB}$, many others causes must be ruled out before giving ATT. However, in the clinical scenario if a patient is diagnosed as granulomatous inflammation, then antituberculous treatment (ATT) is started at the first point in our setup. Statistically this behavior may be right but this is not in accordance with the reality. We come to encounter cases which have taken ATT for at least 9 months but still these symptoms persist. Reassessment is done, and later the patient is diagnosed as suffering from fungus, sarcoidosis, or some other granulomatous disease. Some special stains are very helpful in this regard, like Gomori Methenamine silver stains (GMS), Giemsa stain, Periodic acid Schiff (PAS), and Zeihl Neelson's stain (ZN stain) [27].

In the present study, granulomatous inflammation consistent with Tuberculosis diagnosed on FNAC will be analyzed using special stains like ZN (Ziehl Neelson's) and GMS (Gomori Methenamine Silver) stains. This will help to confirm tuberculosis in cases which will be positive for Acid Fast Bacilli on ZN staining. Positive GMS/PAS staining will confirm in the fungal causes of granulomatous inflammation including Mucormycosis, Blastomycosis, Cryptococcosis, and Candidiasis. There is a limitation of this study that not all causes of granulomatous inflammation can be ruled out since the ancillary investigations needed to diagnose them are not available in our setup.

The rationale of this study is that cases diagnosed wrongly as TB can turn out to be fungus and can be picked by GMS/PAS. These patients can thus be saved from long painful and harmful side effects of expensive ATT (Antituberculous therapy). This benefit alone is worth mentioning for the usefulness of this study, and this would be further reaffirmed by those who have experienced taking ATT for 9 months in their life without having TB. On ZN staining, the positive AFB cases would help the physicians to start treatment of TB, very confidently. Moreover, the work done to assess the frequency of different infectious agents in granulomatous inflammation especially fungal causes is very old, and this study would bridge a gap between newer studies done on this topic.

\section{Material and Methods}

2.1. Setting. The study was conducted at Pathology Department of King Edward Medical University and Mayo Hospital Lahore. The department receives 10,000 surgical specimens and 3000 cytology specimens including FNAC annually.

\subsection{Duration. Six months.}

2.3. Sample Size. Sample size of 100 cases was calculated with $95 \%$ confidence level, $8 \%$ margin of error, and taking expected percentage of positive cases of fungus on GMS/PAS that is, $20.4 \%$ in diagnosed cases of granulomatous inflammation.

2.4. Sampling Technique. Nonprobability purposive sampling.

\subsection{Inclusion Criteria}

(1) Cases diagnosed on FNAC as granulomatous inflammation consistent with tuberculosis as per operational definitions.

(2) Cases in which FNAC was done on Lymph node, skin swellings, subcutaneous swellings, and Lung masses

\subsection{Exclusion Criteria}

(1) Pyogenic inflammation seen on microscopy as extensive neutrophilic infiltration.

(2) Acellular smears/smears with crushed morphology or poorly stained slides will be excluded.

(3) Previously diagnosed cases and cases already getting ATT.

\subsection{Study Design. Descriptive cross-sectional survey.}

\subsection{Operational Definitions}

2.8.1. Granulomatous Inflammation. It is defined on cytology as aggregates of epitheloid cells forming a granuloma with or without necrosis. Sometimes multinucleated giant cells are also seen.

2.8.2. Positive for $A F B$. On $\mathrm{ZN}$ staining the acid fast bacilli would be labeled when we find pink, beaded, and rod-shaped organisms after comparing with control samples.

2.8.3. Positive for Fungus. On GMS staining, presence of black colored septated or nonseptated hyphae (depending upon the species of Fungus) or spores against a greenish background would be labeled as positive for fungus. On PAS stain, presence of red- or purple-colored septated or nonseptated hyphae or spores would be labeled as positive for fungus.

2.9. Data Collection Procedure. Patients fulfilling inclusion and exclusion criterion were selected from Fine needle aspiration cytology specimens received during the study period. After informed consent of patients and noting down the demographic data, the hematoxylin and Eosin staining was done. Two extra unstained slides were smeared from aspiration material. One slide was stained by GMS stain. Some cases were stained with Periodic Acid Schiff stain (PAS). Steps of PAS staining are as follows: similarly 2nd unstained slide was stained with Ziehl Nelson's stain. Commercially available positive and negative controls of $\mathrm{ZN}$ 
TABLE 1: Distribution of age of patients.

\begin{tabular}{lcc}
\hline Age of patient (years) & $\begin{array}{c}\text { Granulomatous } \\
\text { inflammation }\end{array}$ & $\begin{array}{c}\text { \% of granulomatous } \\
\text { inflammation }\end{array}$ \\
\hline $1-10$ & 3 & 3 \\
$11-20$ & 44 & 44 \\
$21-30$ & 31 & 31 \\
$31-40$ & 11 & 11 \\
$41-50$ & 8 & 8 \\
$51-60$ & 2 & 2 \\
$61-70$ & 0 & 0 \\
$71-80$ & 1 & 1 \\
Total mean $=25.14 \pm$ & 100 & 100 \\
12.75 & & \\
\hline
\end{tabular}

TABLE 2: Distribution of gender of patients.

\begin{tabular}{lcc}
\hline Gender & Frequency & Percent $(\%)$ \\
\hline Female & 62 & 62 \\
Male & 38 & 38 \\
\hline Total & 100 & 100 \\
\hline
\end{tabular}

and GMS were used to compare and measure the consistency of staining technique. These smears were examined under the light microscope by a histopathology's. The findings of Hematoxylin and eosin staining were categorized as epitheloid granuloma with necrosis and epitheloid granuloma without necrosis. The finding of $\mathrm{ZN}$ staining was labeled as positive for AFB or negative for AFB. The finding of GMS was recorded as positive for fungus or negative for fungus.

2.10. Data Analysis. Data was analyzed by SPSS version 10 . Age of patient was presented as mean and standard deviation. Gender, positive cases of AFB, and positive cases of fungus were presented as frequency and percentages.

\section{Results}

One hundred patients of granulomatous inflammation diagnosed on FNAC were taken. Granulomas were described as comprising of pale staining epithelioid cells which were round to oval to spindle against an eosinophilic background (Figure 1). Few degenerated epithelioid histiocytes were also seen in long-standing mycobacterial infection with caseation necrosis in the background (Figure 2). On Ziehl Neelson's staining, mycobacterium tuberculosis appeared as red/pink beaded rod-shaped bacteria against a blue background (Figures 2(a) and 2(b)). On PAS staining, fungus appears as purple hyphae which were segmented or nonsegmented depending on the species. Few spore forms with budding were also seen (Figure 2). On GMS stain, fungal hyphen appeared as black-colored forms which showed segmentation and some were nonsegmented (Figure 2).

In this study, $78 \%$ patients were below 30 years of age (Table 1). Mean age was 25.14 with standard deviation of 12.745 . Females were affected more $(68 \%)$ than males
TABLE 3: Frequency of positive smears of acid fast bacilli.

\begin{tabular}{lcc}
\hline Acid fast bacilli & Frequency & Percent \\
\hline Positive for AFB & 44 & 44 \\
Negative for AFB & 56 & 56 \\
\hline Total & 100 & 100
\end{tabular}

TABLE 4: Frequency of fungus and granulomatous inflammation.

\begin{tabular}{lcc}
\hline Fungus & Frequency & Percent \\
\hline Negative for fungus & 95 & 95 \\
Positive for fungus & 05 & 05 \\
\hline Total & 100 & 100 \\
\hline
\end{tabular}

(Table 2). 44 out of 100 patients of granulomatous inflammation are positive for AFB (Table 3). There was an association between AFB positivity and caseation necrosis. We have found 41 out of total 44 AFB positive cases (93\%) with caseation necrosis (Table 6), while $60 \%$ cases of fungus were related to caseation (Table 7). No definite relationship was seen between AFB and giant cells since 19 out of total $44 \mathrm{AFB}$ positive cases were seen with caseation while rest $57 \%$ were without giant cells. Another finding was the involvement of specific lymph nodes regions. In $87 \%$ of cases, the most commonly involved group of lymph nodes was cervical lymph node (combining cervical and supraclavicular lymph nodes). If per auricular lymph nodes were included, then in $93 \%$ of cases the head and neck was the primary site of TB involvement (Table 5).

\section{Discussion}

Accurate and timely diagnosis together with effective TB treatment is the mainstay of $\mathrm{TB}$ care and control. A confirmed diagnosis of $\mathrm{TB}$ can only be given on isolating the $\mathrm{M}$. tuberculosis or finding specific DNA sequence of the bacteria in aspirates. In the resource-poor countries, however, these tests are not within the reach of every individual. In these countries, cost-effective techniques for example, sputum smear microscopy and morphological features are the corner stone of TB diagnosis. In cases of extra pulmonary tuberculosis, fine needle aspiration cytology (FNAC) is a very useful and reliable test. In areas where tuberculosis is prevalent, diagnosis of $\mathrm{TB}$ can be made by seeing the morphological features. Granulomatous inflammation is the common histological presentation of tuberculosis. However, there are many other infectious and noninfectious causes which can lead to granulomatous inflammation. Second important infectious cause of granulomatous inflammation is fungus. In the present study, we tried to differentiate between granulomatous inflammation caused by TB and fungus, by using special stains.

Blind FNAC can approach safely the superficial lesions, including lymph nodes, skin, and soft tissue nodules. In our study, 98 (98\%) cases were from lymph nodes. Many studies have diagnosed TB by aspiration form lymph nodes $[7,11,26,28,29]$. Cervical lymph node was the most 
TABLE 5: Distribution lesions according to site of FNAC.

\begin{tabular}{lcccc}
\hline Site of FNAC & Frequency & Percent & Valid percent & Cumulative percent \\
\hline Cervical lymph node & 72 & 72.0 & 72.0 & 72.0 \\
Peri-auricular lymph nodes & 6 & 6.0 & 6.0 & 78.0 \\
Supraclavicular lymph node & 15 & 15.0 & 15.0 & 93.0 \\
Axillary lymph node & 3 & 3.0 & 3.0 & 96.0 \\
Inguinal lymph node & 2 & 2.0 & 2.0 & 98.0 \\
Skin or subcutaneous lesion & 1 & 1.0 & 1.0 & 99.0 \\
Other sites & 1 & 1.0 & 1.0 & 100.0 \\
\hline Total & 100 & 100.0 & 100.0 &
\end{tabular}

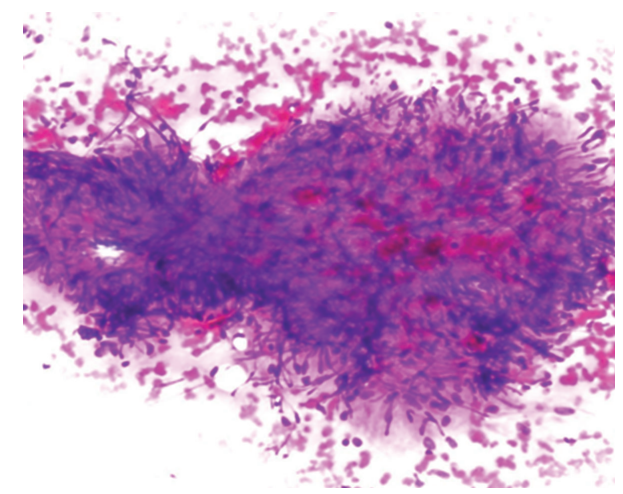

(a)

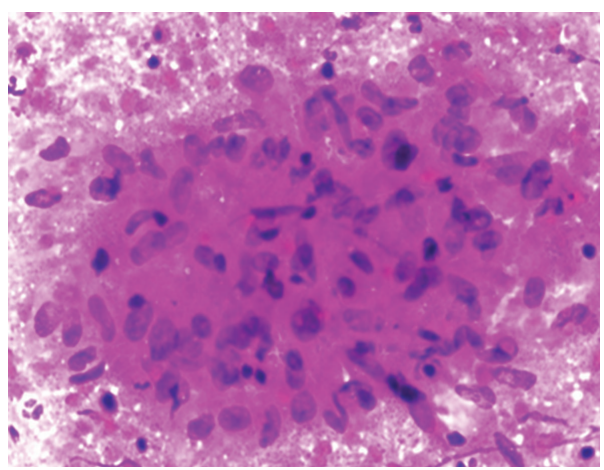

(c)

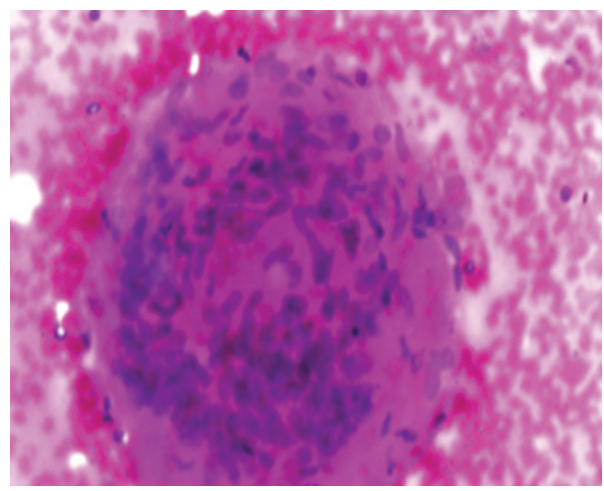

(b)

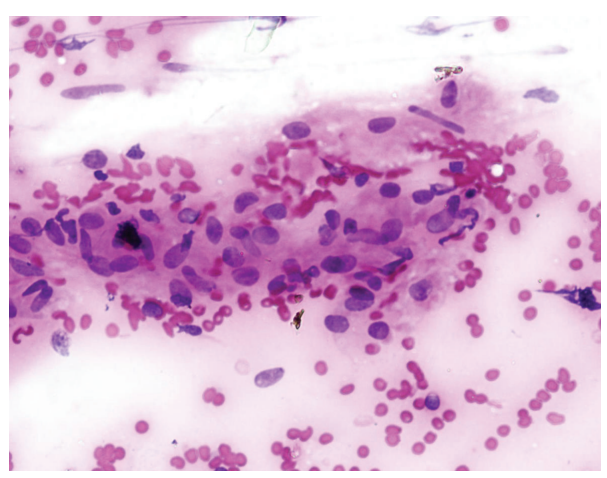

(d)

Figure 1: (a) Photomicrograph showing degenerated granulomas with caseation necrosis (H \& E stain, 100x), (b) Granulomatous inflammation on FNAC (H \& E stain, 200x) giant cells, (c) Granulomas comprising of epithelioid histiocytes with caseation necrosis (H \& E stain, 200x), (d) Aggregates of pale staining epithelioid histiocytes (H \& E, 400x).

TABLE 6: Relationship of acid fast bacilli with caseation necrosis.

\begin{tabular}{lccc}
\hline & \multicolumn{2}{c}{ Acid fast bacilli } & Total \\
& $\begin{array}{c}\text { negative for } \\
\text { AFB }\end{array}$ & $\begin{array}{c}\text { positive for } \\
\text { AFB }\end{array}$ & \\
\hline Caseation necrosis & 28 & 3 & 31 \\
Not present & 28 & 41 & 69 \\
$\quad$ Present & 56 & 44 & 100 \\
\hline Total & & & \\
\hline
\end{tabular}

TABLE 7: Frequency of caseation necrosis with fungus.

\begin{tabular}{lccc}
\hline & \multicolumn{2}{c}{ Fungus } & Total \\
& $\begin{array}{c}\text { negative for } \\
\text { fungus }\end{array}$ & $\begin{array}{c}\text { positive for } \\
\text { fungus }\end{array}$ & \\
\hline Caseation necrosis & 29 & 2 & 31 \\
$\quad$ Not present & 66 & 3 & 69 \\
$\quad$ Present & 95 & 5 & 100 \\
\hline Total & & & \\
\hline
\end{tabular}




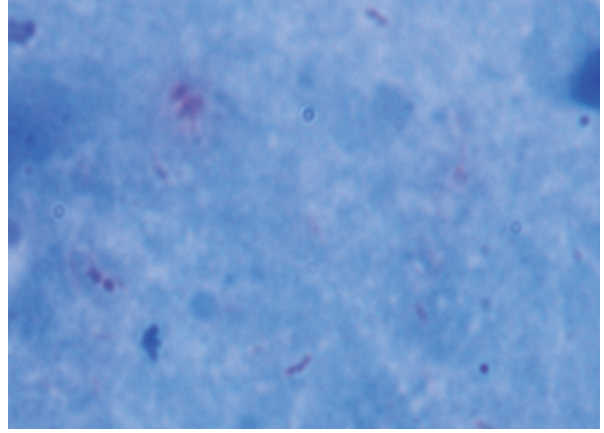

(a)

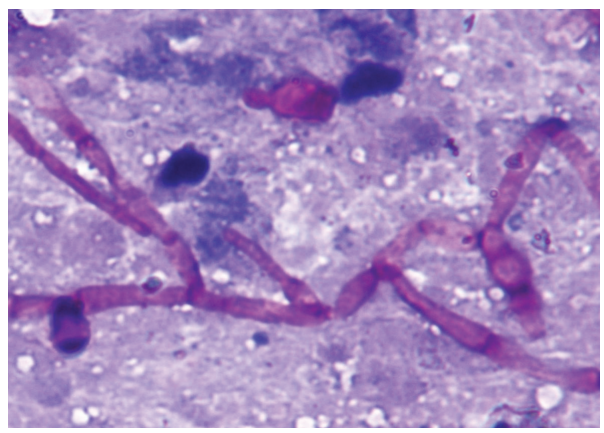

(c)

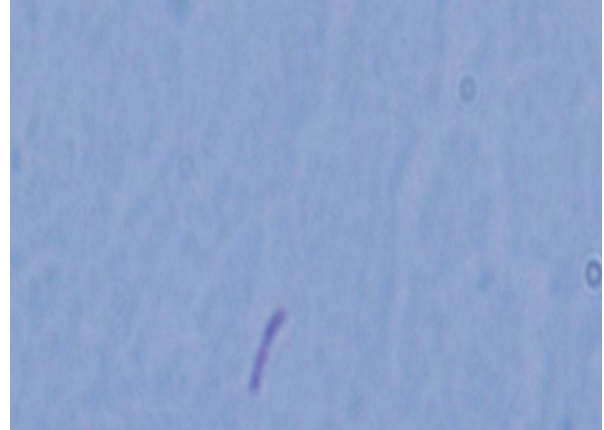

(b)

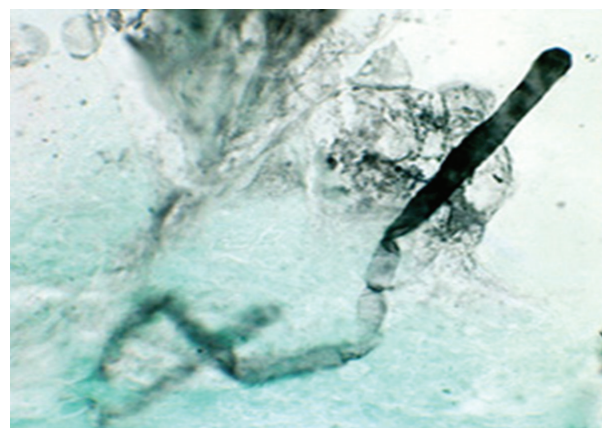

(d)

FIgURE 2: Photomicrographs in (a) Acid Fast Bacilli (Ziehl Nelson's stain, 400x), (b) Pink beaded rod against a blue background (ZN stain, 1000x), (c) Septated fungal hyphae and budding spore (at the top of photomicrograph), (PAS, 400x), (d) Black fungal hyphae against greenish background (GMS, 400x).

TABLE 8: Frequency of acid fast bacilli with giant cells.

\begin{tabular}{lccc}
\hline & \multicolumn{2}{c}{ Acid fast bacilli } & Total \\
& $\begin{array}{c}\text { negative for } \\
\text { AFB }\end{array}$ & $\begin{array}{c}\text { positive } \\
\text { for AFB }\end{array}$ & \\
\hline Giant cells & & & \\
Not present & 50 & 25 & 75 \\
$\quad$ Present & 6 & 19 & 25 \\
\hline Total & 56 & 44 & 100 \\
\hline
\end{tabular}

common site of involvement in studies followed by axillary lymph nodes $[11,21]$. Our study was also consistent with above studies in terms of cervical lymph node involvement $(87 \%)$ as the most common anatomic site of granulomatous inflammation. Periauricular lymph nodes were involved in $6 \%$ cases in our study and was the second most commonly involved. Female gender was a slightly more affected (62\%) in current study and was in concordance with other studies [26]. However, there was slight male predominance in a study of Bezabih et al. [11]. Out of 100, 47\% patients in this study were of 20 years or below and $62 \%$ were below 30 . This finding was in accordance with Bezabih et al. in which $69 \%$ were below 30. Based on the facts, it can be inferred that tuberculosis was more commonly seen in young population [11].

One case of granulomatous inflammation was from skin (Table 5). Few studies from India have also discussed this aspect [30]. Numerous morphological variations in the granulomatous inflammation are seen. There were $69 \%$ cases with necrosis. The rest (31\%) of cases were granulomatous inflammation without necrosis. The various morphological presentations of TB have been published locally [7]. International data also supports this variation and studies tried to correlate morphological findings with the AFB staining $[11,31]$.

The Acid Fast Bacilli positivity was labeled after finding red or pink rod-shaped bacteria with beaded appearance (Figures 2(a) and 2(b)). Regarding AFB positivity variable, results were seen and frequency ranges from $10 \%$ to $70 \%$ [26, 27, 30, 31].

In current study, out of 100 cases, 44 cases were positive for AFB (44\%). This was in concordance with the international data of a large-scale study of 328 cases, out of which 152 cases (46.4\%) were positive for AFB [21]. Similarly, our findings agree with Lau et al. who report $47 \%$ sensitivity for tuberculous abscess cases [26] and with Das et al. showing overall 45.8\% rate of AFB positivity [32]. A study conducted in India shows an overall $27 \%$ AFB positivity [31], and the reason for this low AFB sensitivity was given: studies with higher AFB have adult subjects, in whom open tuberculosis and necrotic lesion were far more common. Examples of low yield of AFB were also due to treatment with antituberculosis drugs and presence of very few bacilli in the lymph node [31]. Some studies report very high frequency of AFB positivity. Bezabih et al. reported $59.4 \%$ of overall AFB positivity [30], and Vignesh et al. reported 53.3\% sensitivity for single AFB smear [27]. 
In regions where $\mathrm{TB}$ is very common, the morphological findings of granulomatous inflammation is consistent with tuberculosis [30, 31]. Pakistan is also included among these countries along with India, Ethiopia, and other African countries. Since epithelioid granulomas, caseation necrosis, giant cells, and AFB positivity are specific for $\mathrm{TB}$, so in these countries excision biopsy can be avoided and antituberculous treatment can be given straightaway [26]. Excision is not free of complication and is expensive and time consuming, thus it can delay the treatment. Above findings conclude that FNAC with special stains can solely help the physician to start the treatment.

There was an interesting finding in our study. AFB positivity was notablely and more commonly found in granulomatous inflammation with caseation necrosis. 41 out of $44 \mathrm{AFB}$ positive cases associated with caseation necrosis $(93 \%)$, in current study. This finding is consistently seen in previous studies $[11,21,31]$. Otherwise in some studies, it is claimed that instead of granulomatous inflammation, if only necrosis or abscess formation is seen, the AFBpositivity increases [26]. Dua et al. even documented $100 \%$ of AFB positive cases in this scenario [31]. Since in the inclusion criterion of our study we only selected cases with granulomatous inflammation with or without necrosis, but not cases only with necrosis, this aspect cannot be discussed in this study. Most of studies improved the technique of finding AFB by using fluorescence microscopy. They claimed at least $10 \%$ improvement in sensitivity and sensitivity if fluorescence microscopy is used as compared to direct smear examination $[33,34]$. However, in resource-poor countries it would still take some time to gain wide acceptance.

Another interesting finding was that an acid fast bacillus was usually found extracellularly. Usually areas of microscopic degeneration, within or at the periphery of the granulomas, were most the common location to find AFB [21]. The morphology of these bacilli was short and stumpy rods with red beaded appearance. These findings correlated with those given by Rajasekaran et al. [35] and Ahmad et al. [21]. For early lesions of tuberculous lymphadenopathy, there is no evidence that chemotherapy (ATT) plus excision is superior than chemotherapy alone [26]. Moreover, the excision biopsy in tuberculous lymph nodes is hazardous since it may cause sinus formation. Therefore, FNAC finding of granulomatous inflammation and detection of AFB would be very specific and help the physicians to start ATT confidently, immediately as it is cost effective and economical.

The special stains GMS and PAS were used to detect the fungus, since it may present with same morphology as $\mathrm{TB}[14,17,36]$. In this study, we found $5 \%$ cases of fungus presenting with granulomatous inflammation. After extensive search of the literature, only one study was found in which $20.4 \%$ cases of fungus occurred among 245 subjects [14]. Yet many other studies discussed fungus as a cause of granulomatous inflammation and published them as case reports [14, 36-39]. But these studies did not mention frequency or percentage of positive case of fungus. In this regard, the present study would bridge a gap and may become a source of future reference for further studies in this aspect. The main benefit we gained from this study was that these patients were diagnosed morphologically as "consistent with tuberculosis". However, the results via special stains established that it can be caused by fungus and not only by mycobacterium tuberculosis. Added benefit is that these patients would be safe from harmful side effects of prolonged ATT treatment. They can get antifungal treatment, and the disease can be cured. In this study, we did not classify species of fungus on these special stains for it may not be accurate. For this purpose, fungal cultures should be performed.

\section{Recommendations}

(1) Every case of granulomatous inflammation seen on aspiration cytology should be subjected to special stains like ZN, GMS/PAS. It would increase the diagnostic accuracy of this technique and help to differentiate between different infectious causes which can present with the same morphology.

(2) When physicians are confronted with enlarged lymph nodes, the node may be punctured with a sterile disposable needle, and if cheesy material is aspirated then the physician can strongly consider tuberculous adenitis in areas where tuberculosis and immunodeficiency states are rampant and pathology services are lacking.

(3) Patients who are not responding to empirical ATT should be considered for other causes of granulomatous inflammation other than $\mathrm{TB}$, and proper workup should be done.

\section{Limitation of Study}

(1) This study does not include comparison with histology and other microbiological detection methods like culture and PCR, because of cost and unavailability issues.

(2) Our study did not comment on all the possible differential diagnosis of granulomatous inflammation, which requires sophisticated techniques and tertiary care laboratory services which are currently not available in our setup.

Future Studies. On the current issue, future studies should include comparison of direct smear microscopy of AFB with fluoroscopic evaluation. Moreover, the aspirate of FNAC should be subjected not only for special stains, but also for immunohistochemical stains, culture and PCR, and then compared for efficacy.

\section{Conclusion}

Fine needle aspiration cytology (FNAC) is very important investigation in the diagnosis of granulomatous inflammation. If it is supplemented with special stains like ZN, GMS, and PAS, it may help to differentiate between many infectious causes of granulomatous inflammation. 


\section{References}

[1] N. Ishikawa, "How to combat against global tuberculosis burden-experiences and perspectives for Japan's international cooperation," Kekkaku, vol. 80, no. 2, pp. 89-94, 2005.

[2] Z. Hasan, M. Tanveer, A. Kanji, Q. Hasan, S. Ghebremichael, and R. Hasan, "Spoligotyping of Mycobacterium tuberculosis isolates from Pakistan reveals predominance of central Asian strain 1 and Beijing isolates," Journal of Clinical Microbiology, vol. 44, no. 5, pp. 1763-1768, 2006.

[3] R. Agarwal, R. Srinivas, and A. N. Aggarwal, "Parenchymal pseudotumoral tuberculosis: case series and systematic review of literature," Respiratory Medicine, vol. 102, no. 3, pp. 382389,2008

[4] P. Nuwal, R. Dixit, S. Jain, and V. Porwal, "Isolated appendicular tuberculosis-a case report," The Indian Journal of Tuberculosis, vol. 47, no. 4, pp. 241-242, 2000.

[5] S. Rasheed, R. Zinicola, D. Watson, A. Bajwa, and P. J. Mcdonald, "Intra-abdominal and gastrointestinal tuberculosis," Colorectal Disease, vol. 9, no. 9, pp. 773-783, 2007.

[6] G. Akoglu, A. Karaduman, G. Boztepe et al., "A case of lupus vulgaris successfully treated with antituberculous therapy despite negative PCR and culture," Dermatology, vol. 211, no. 3, pp. 290-292, 2007.

[7] A. Sarwar, S. Aftab, M. Mustafa, A. Moatasim, S. Siddique, and A. Sani, "Spectrum of morphological changes in tuberculous lymphadenitis," Internation journal of Pathology, vol. 2, no. 2, pp. 85-89, 2004.

[8] S. Nayak and R. Satish, "Genitourinary tuberculosis after renal transplantation-a report of three cases with a good clinical outcome," American Journal of Transplantation, vol. 7, no. 7, pp. 1862-1864, 2007.

[9] R. B. Rock, M. Olin, C. A. Baker, T. W. Molitor, and P. K. Peterson, "Central nervous system tuberculosis: pathogenesis and clinical aspects," Clinical Microbiology Reviews, vol. 21, no. 2, pp. 243-261, 2008.

[10] S. K. Sinha, M. Chatterjee, S. Bhattacharya et al., "Diagnostic evaluation of extrapulmonary tuberculosis by fine needle aspiraton (FNA) supplemented with AFB smear and culture," Journal of the Indian Medical Association, vol. 101, no. 10, pp. 590-591, 2003.

[11] M. Bezabih, D. W. Mariam, and S. G. Selassie, "Fine needle aspiration cytology of suspected tuberculous lymphadenitis," Cytopathology, vol. 13, no. 5, pp. 284-290, 2002.

[12] S. M. Lam, A. C. W. Lau, M. W. Ma, and L. Y. C. Yam, "Pseudallescheria boydii or Aspergillus fumigatus in a lady with an unresolving lung infiltrate, and a literature review," Respirology, vol. 13, no. 3, pp. 478-480, 2008.

[13] S. Mamishi, N. Parvaneh, A. Salavati, S. Abdollahzadeh, and M. Yeganeh, "Invasive aspergillosis in chronic granulomatous disease: report of 7 cases," European Journal of Pediatrics, vol. 166, no. 1, pp. 83-84, 2007.

[14] M. S. Cohen, R. E. Isturiz, H. L. Malech et al., "Fungal infection in chronic granulomatous disease. The importance of the phagocyte in defense against fungi," American Journal of Medicine, vol. 71, no. 1, pp. 59-66, 1981.

[15] J. S. Klein, A. Johnson, E. Watson, and S. Mount, "CT-guided transthoracic needle biopsy in the diagnosis of sarcoidosis," Journal of Thoracic Imaging, vol. 24, no. 1, pp. 23-30, 2009.

[16] A. Mueller, K. Holl-Ulrich, P. Lamprecht, and W. L. Gross, "Germinal centre-like structures in Wegener's granuloma: the morphological basis for autoimmunity?" Rheumatology, vol. 47 , no. 8, pp. 1111-1113, 2008.
[17] V. Mehta and C. Balachandran, "Primary cutaneous actinomycosis on the chest wall," Dermatology Online Journal, vol. 14, no. 8, article no. 13, 2008.

[18] Y. Al-Gindan, M. Satti, A. Al-Quorain, and A. Al-Hamdan, "Crohn's disease in Saudi Arabia: A clinicopathological study of 12 cases," Saudi Journal of Gastroenterology, vol. 2, no. 3, p. $150,2010$.

[19] H. V. K. Naina, C. F. Thomas, and S. Harris, "Histoplasmosis and asplenia," Thorax, vol. 65, no. 4, p. 372, 2010.

[20] H. W. Tan, K. L. Chuah, S. G. N. Goh, W. M. Yap, and P. H. Tan, "An unusual cause of granulomatous inflammation: eosinophilic abscess in Langerhans cell histiocytosis," Journal of Clinical Pathology, vol. 59, no. 5, pp. 548-549, 2006.

[21] S. S. Ahmad, S. Akhtar, K. Akhtar, S. Naseem, and T. Mansoor, "Study of fine needle aspiration cytology in lymphadenopathy with special reference to acid-fast staining in cases of tuberculosis," JK Science, vol. 7, no. 1, pp. 1-4, 2005.

[22] S. Chakravorty, M. K. Sen, and J. S. Tyagi, "Diagnosis of extrapulmonary tuberculosis by smear, culture, and PCR using universal sample processing technology," Journal of Clinical Microbiology, vol. 43, no. 9, pp. 4357-4362, 2005.

[23] K. E. Volmar, H. K. Singh, and J. Z. Gong, "Fine-needle aspiration biopsy of lymph nodes in the modern era: reactive lymphadenopathies," Pathology Case Reviews, vol. 12, no. 1, pp. 27-35, 2007.

[24] S. Mirza, B. I. Restrepo, J. B. McCormick, and S. P. FisherHoch, "Diagnosis of tuberculosis lymphadenitis using a polymerase chain reaction on peripheral blood mononuclear cells," American Journal of Tropical Medicine and Hygiene, vol. 69, no. 5, pp. 461-465, 2003.

[25] M. Saha, M. Bakar, M. Rahman, and S. Hossain, "Role of FNA cytology in the diagnosis of lymph node diseases," Journal of Bangladesh College of Physicians and Surgeons, vol. 23, no. 1, p. 30, 2005.

[26] S. K. Lau, W. U. Wei, C. Hsu, and U. C. G. Engzell, "Efficacy of fine needle aspiration cytology in the diagnosis of tuberculous cervical lymphadenopathy," Journal of Laryngology and Otology, vol. 104, no. 1, pp. 24-27, 1990.

[27] R. Vignesh, P. Balakrishnan, E. M. Shankar et al., "Value of single acid-fast bacilli sputum smears in the diagnosis of tuberculosis in HIV-positive subjects," Journal of Medical Microbiology, vol. 56, no. 12, pp. 1709-1710, 2007.

[28] V. Koo, T. F. Lioe, and R. A. J. Spence, "Fine needle aspiration cytology (FNAC) in the diagnosis of granulomatous lymphadenitis," Ulster Medical Journal, vol. 75, no. 1, pp. 5964, 2006.

[29] A. Rajwanshi, S. Bhambhani, and D. K. Das, "Fine-needle aspiration cytology diagnosis of tuberculosis," Diagnostic Cytopathology, vol. 3, no. 1, pp. 13-16, 1987.

[30] G. Sethuraman, V. Ramesh, M. Ramam, and V. K. Sharma, "Skin tuberculosis in children: learning from India," Dermatologic Clinics, vol. 26, no. 2, pp. 285-294, 2008.

[31] T. Dua, P. Ahmad, S. Vasenwala, F. Beg, and A. Malik, "Correlation of cytomorphology with AFB positivity by smear and culture in tuberculous lymphadenitis," The Indian Journal of Tuberculosis, vol. 43, pp. 81-84, 1996.

[32] D. K. Das, C. S. Pant, J. N. Pant, and P. Sodhani, "Transthoracic (percutaneous) fine needle aspiration cytology diagnosis of pulmonary tuberculosis," Tubercle and Lung Disease, vol. 76, no. 1, pp. 84-89, 1995.

[33] K. R. Steingart, M. Henry, V. Ng et al., "Fluorescence versus conventional sputum smear microscopy for tuberculosis: a systematic review," The Lancet Infectious Diseases, vol. 6, no. 9, pp. 570-581, 2006. 
[34] M. R. Nyendak, D. A. Lewinsohn, and D. M. Lewinsohn, "New diagnostic methods for tuberculosis," Current Opinion in Infectious Diseases, vol. 22, no. 2, pp. 174-182, 2009.

[35] S. Rajasekaran, M. Gunasekaran, D. Jayakumar, D. Jeyaganesh, and V. Bhanumati, "Tuberculous cervical lymphadenitis in HIV positive and negative patients," The Indian Journal of Tuberculosis, vol. 48, no. 4, pp. 201-204, 2001.

[36] S. Shukla, S. Singh, M. Jain, S. K. Singh, R. Chander, and N. Kawatra, "Paediatric cutaneous blastomycosis: a rare case diagnosed on FNAC," Diagnostic Cytopathology, vol. 37, no. 2, pp. 119-121, 2009.

[37] S. Suchitha, R. Sunila, and G. V. Manjunath, "Fine needle aspiration diagnosis of cryptococcal lymphadenitis: a window of opportunity," Journal of Cytology, vol. 25, no. 4, pp. 147149, 2008.

[38] B. R. J. Shravanakumar, K. R. Iyengar, Y. Parasappa, and R. Ramprakash, "Cryptococcal lymphadenitis diagnosed by FNAC in a HIV positive individual," Journal of Postgraduate Medicine, vol. 49, no. 4, p. 370, 2003.

[39] Y. M. Al-Marzooq, R. Chopra, M. I. Al-Mommatten, M. Younis, A. S. Al-Mulhim, and H. Al-Hussini, "Fine-needle aspiration diagnosis of primary cutaneous cryptococcosis in an immunocompetent patient: a case report," Diagnostic Cytopathology, vol. 32, no. 4, pp. 219-221, 2005. 


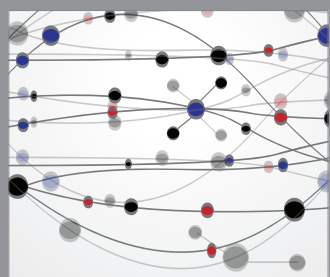

The Scientific World Journal
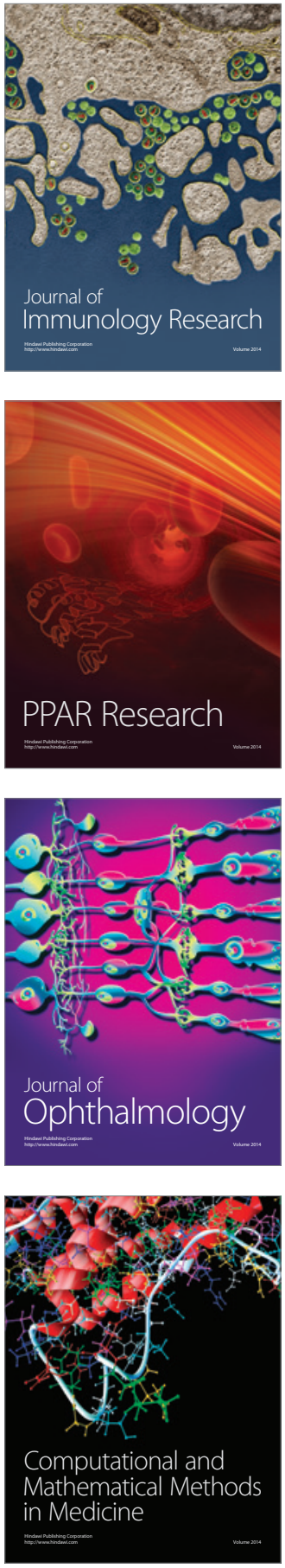

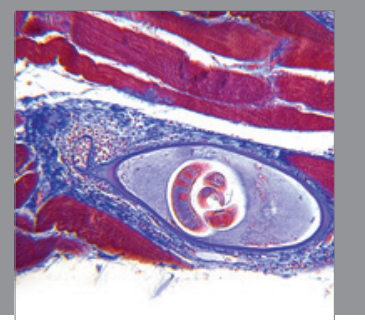

Gastroenterology

Research and Practice
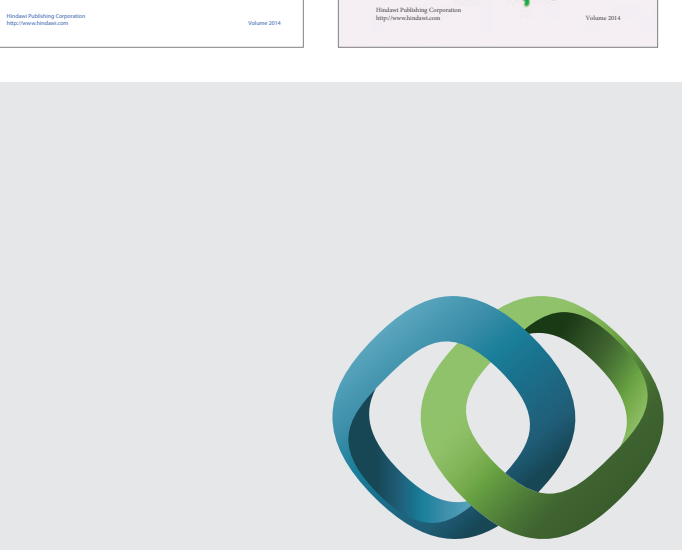

\section{Hindawi}

Submit your manuscripts at

http://www.hindawi.com
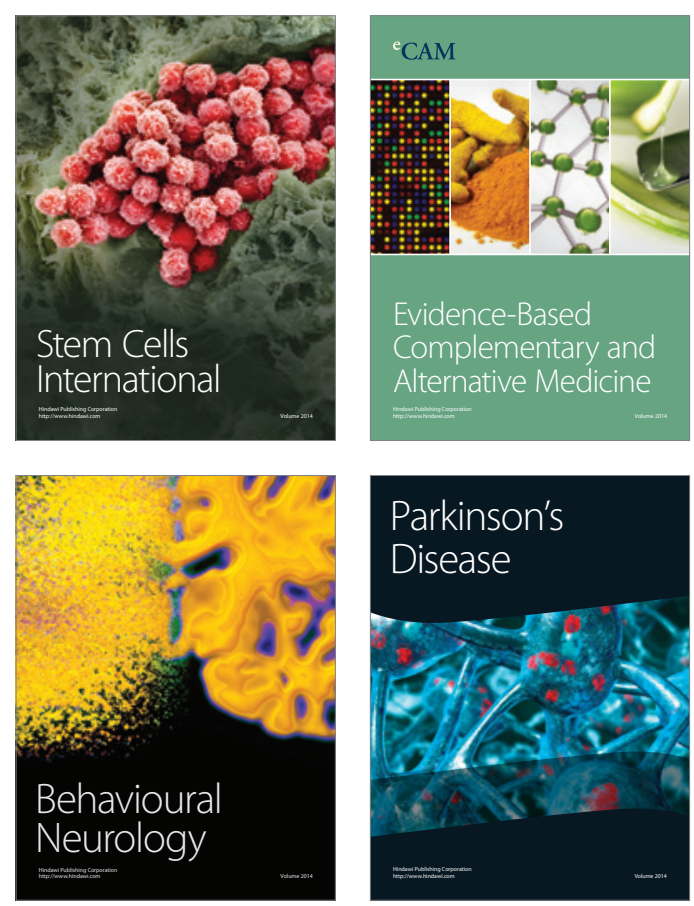

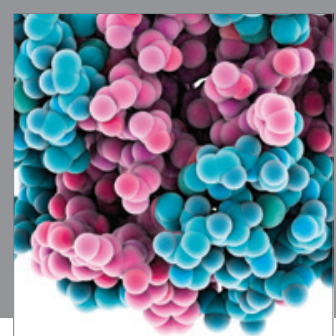

Journal of
Diabetes Research

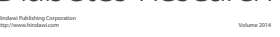

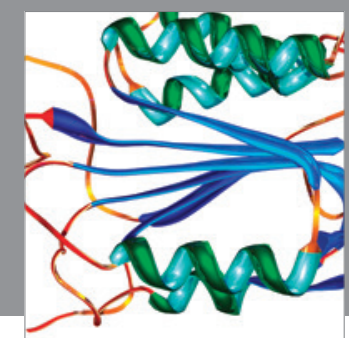

Disease Markers
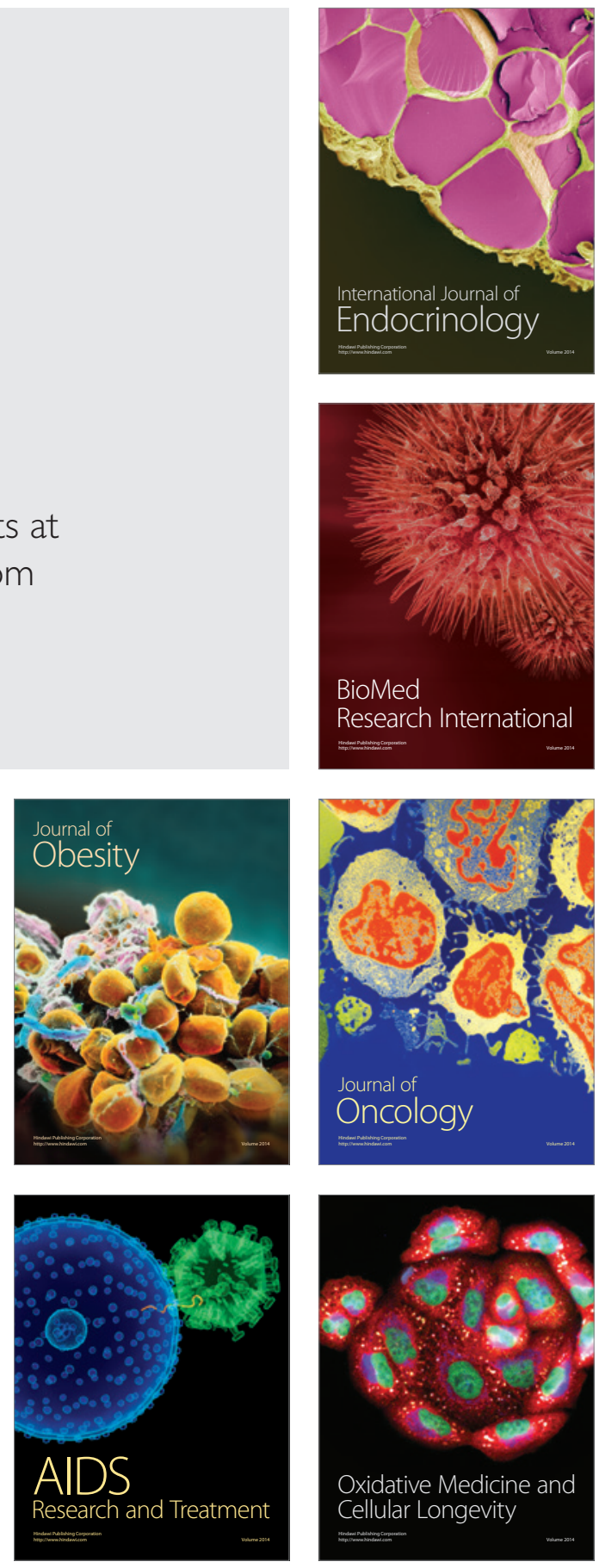